\title{
PERTURBATION OF EIGENVALUES OF PRECONDITIONED NAVIER-STOKES OPERATORS
}

\author{
HOWARD C. ELMAN * \\ Report CS-TR-3559 \\ UMIACS-TR-95-110 \\ November 1995 \\ Revised July 1996
}

\begin{abstract}
We study the sensitivity of algebraic eigenvalue problems associated with matrices arising from linearization and discretization of the steady-state Navier-Stokes equations. In particular, for several choices of preconditioners applied to the system of discrete equations, we derive upper bounds on perturbations of eigenvalues as functions of the viscosity and discretization mesh size. The bounds suggest that the sensitivity of the eigenvalues is at worst linear in the inverse of the viscosity and quadratic in the inverse of the mesh size, and that scaling can be used to decrease the sensitivity in some cases. Experimental results supplement these results and confirm the relatively mild dependence on viscosity. They also indicate a dependence on the mesh size of magnitude smaller than the analysis suggests.
\end{abstract}

\footnotetext{
* Department of Computer Science and Institute for Advanced Computer Studies, University of Maryland, College Park, MD 20742, e-mail: elman@cs.umd.edu. This work was supported by the U.S. Army Research Office under grant DAAL-0392-G-0016 and the U. S. National Science Foundation under grants ASC-8958544 and DMS-9423133.
} 


\title{
PERTURBATION OF EIGENVALUES OF PRECONDITIONED NAVIER-STOKES OPERATORS
}

\author{
HOWARD C. ELMAN *
}

\begin{abstract}
We study the sensitivity of algebraic eigenvalue problems associated with matrices arising from linearization and discretization of the steady-state Navier-Stokes equations. In particular, for several choices of preconditioners applied to the system of discrete equations, we derive upper bounds on perturbations of eigenvalues as functions of the viscosity and discretization mesh size. The bounds suggest that the sensitivity of the eigenvalues is at worst linear in the inverse of the viscosity and quadratic in the inverse of the mesh size, and that scaling can be used to decrease the sensitivity in some cases. Experimental results supplement these results and confirm the relatively mild dependence on viscosity. They also indicate a dependence on the mesh size of magnitude smaller than the analysis suggests.
\end{abstract}

Key words. eigenvalues, perturbation analysis, Navier-Stokes, preconditioning.

AMS(MOS) subject classifications. Primary: 65F10, 65N20; secondary 15A06

1. Introduction. This paper concerns properties of the eigenvalues of matrices arising from the discrete linearized steady-state Navier-Stokes equations. The continuous problem is

$$
-\nu \Delta \mathbf{u}+(\mathbf{u} \cdot \operatorname{grad}) \mathbf{u}+\operatorname{grad} p=\mathbf{f} \quad \text { in } \Omega,
$$

together with the incompressibility constraint

$$
-\operatorname{div} \mathbf{u}=0 \quad \text { in } \Omega \text {, }
$$

subject to suitable boundary conditions on $\partial \Omega$, where $\Omega$ is an open bounded domain in $\mathbf{R}^{2}$. These equations constitute a fundamental problem in computational fluid dynamics, see e.g., [1],[6],[8]. The two-dimensional vector field u represents the velocity in $\Omega, p$ represents pressure, and the scalar $\nu$ is the viscosity, roughly speaking, the ratio of convection to diffusion in the system.

A methodology for computing the numerical solution is to discretize (1)-(2) using finite difference or finite element methods, and then to solve the resulting nonlinear system by some iterative method. Linearization leads to a set of matrix equations of the form

$$
\left(\begin{array}{cc}
F & B^{T} \\
B & 0
\end{array}\right)\left(\begin{array}{l}
\mathbf{u} \\
p
\end{array}\right)=\left(\begin{array}{l}
\mathbf{f} \\
0
\end{array}\right),
$$

where $\mathbf{u}$ and $p$ now represent discrete versions of velocity and pressure, respectively.

We will restrict our attention to the discrete Oseen equations

$$
\begin{aligned}
& -\nu \Delta \mathbf{u}+(\mathbf{w} \cdot \operatorname{grad}) \mathbf{u}+\operatorname{grad} p=\mathbf{f} \\
& -\operatorname{div} \mathbf{u}=0
\end{aligned}
$$

* Department of Computer Science and Institute for Advanced Computer Studies, University of Maryland, College Park, MD 20742, e-mail: elman@cs.umd.edu. This work was supported by the U.S. Army Research Office under grant DAAL-0392-G-0016 and the U. S. National Science Foundation under grants ASC-8958544 and DMS-9423133. 
where $\mathbf{w}$ is given such that $\operatorname{div} \mathbf{w}=0$. These equations arise from a nonlinear iteration of the form $-\nu \Delta \mathbf{u}^{(m)}+\left(\mathbf{u}^{(m-1)} \cdot \operatorname{grad}\right) \mathbf{u}^{(m)}+\operatorname{grad} p^{(m)}=\mathbf{f},-\operatorname{div} \mathbf{u}^{(m)}=0$, see [10]. In this case

$$
F=\nu A+N
$$

where $A$ consists of a pair of uncoupled discrete Laplace operators, corresponding to diffusion, and $N$ is a skew-symmetric matrix representing convection. We will also assume that the velocity and pressure discretizations are div-stable; see, e.g., [1], p. $57,[8]$, pp. 10ff, [16]. In matrix notation, this is equivalent to the condition

$$
\gamma^{2} \leq \frac{\left(p, B A^{-1} B^{T} p\right)}{(p, M p)} \leq \Gamma^{2} \quad \text { for all } p,
$$

where $(\cdot, \cdot)$ denotes the Euclidean inner product, $\gamma$ and $\Gamma$ are constants that are independent of the discretization mesh size $h$, and for finite elements $M$ is the pressure mass matrix, i.e., the Grammian matrix of basis functions defining the discrete pressure space. ${ }^{1}$ For finite differences on uniform grids, a natural analogue is $M=h^{2} I$.

Let $\mathcal{L}$ denote the coefficient matrix of $(3)$. The following preconditioning matrices were introduced in [3]: a block diagonal preconditioner

$$
\mathcal{Q}_{D}=\left(\begin{array}{cc}
F & 0 \\
0 & \frac{1}{\nu} M
\end{array}\right)
$$

and a block triangular preconditioner

$$
\mathcal{Q}_{T}=\left(\begin{array}{cc}
F & B^{T} \\
0 & -\frac{1}{\nu} M
\end{array}\right)
$$

It was shown in [3] that the eigenvalues of each of the preconditioned matrices $\mathcal{A}_{D}=$ $\mathcal{L} \mathcal{Q}_{D}^{-1}$ and $\mathcal{A}_{T}=\mathcal{L} \mathcal{Q}_{T}^{-1}$ are uniformly bounded independent of the mesh size used in the discretization. Numerical experiments also suggested that Krylov subspace iterative methods such as the generalized minimal residual (GMRES) [13] and quasiminimal residual (QMR) methods [5] can be used to solve the preconditioned system with iteration counts independent of the mesh size.

We are concerned with the sensitivity of the eigenvalues of the preconditioned Oseen matrix using the two preconditioners (6) and (7). Motivation for studying this lies in the fact that use of either preconditioner in an iteration entails applying the action of the inverse of the matrix of either (6) or (7) to a vector at each step. These in turn require the computation of the action of $F^{-1}$, which, if direct methods are used, will dominate the cost. An alternative that was considered in [3] is to approximate the action of $F^{-1}$ (i.e., compute an approximate solution to systems with coefficient matrix $F$ ) using an inner iteration. Unless very stringent stopping criteria are used here, the resulting preconditioned operators can be viewed as perturbations of those of $(6)-(7)$. Thus, we are interested the sensitivity of the eigenvalues to perturbation.

If the preconditioned matrix is perturbed by matrix of size $\epsilon$, then the perturbations of eigenvalues will depend on $\epsilon$ and also on parameters associated with the

\footnotetext{
${ }^{1}$ An inequality analogous to (5) also holds, with different constants, if $M$ is any matrix spectrally equivalent to the mass matrix; for example, $M$ could be the diagonal matrix consisting of the diagonal of the mass matrix [17]. In the sequel, we will not distinguish among such possibilities for $M$.
} 
underlying problem, specifically, the viscosity $\nu$ and mesh size $h$. In this paper, we examine these dependencies using a combination of analytic and experimental results. The analysis derives from Wilkinson's classical perturbation analysis [18], which shows that if there are no nonlinear elementary divisors, the perturbations are of magnitude $O(\epsilon)$. The analytic bounds are stated as functions of $\nu$ and $h$ but they also depend on properties of certain matrices associated with the Schur complement $B F^{-1} B^{T}$ derived from (4). The latter quantities are studied in a series of numerical experiments. The combination of analytic and experimental results indicates that there is an increase in sensitivity to perturbation as the viscosity decreases, with growth roughly linear in $1 / \nu$. This effect can be mitigated to some extent by scaling the first equation of (4) (the momentum equation). The bounds also establish linear dependence on $1 / h$ with the preconditioner $\mathcal{Q}_{T}$ and quadratic dependence with $\mathcal{Q}_{D}$, although the experimental results suggest that perturbations are considerably less sensitive to this parameter.

An outline of the paper is as follows. In $\S 2$, we derive preliminary bounds and relations for several operators associated with the preconditioned matrices. In $\S 3$, we derive the analytic perturbation bounds for the block tridiagonal preconditioner, and in $\S 4$, we present the analysis for the block diagonal preconditioner. For simplicity, the analysis is done for the case where the coefficient matrix of (4) has full rank, although often in practice (and in our experiments) it is rank deficient by one because the pressure $p$ is uniquely defined only up to a constant. In $\S 5$, we show that the analytic results carry over to this case. In $\S 6$, we present the experimental results, and in $\S 7$, we show how the analysis applies for the case of inexact computation of the action of $F^{-1}$.

2. Preliminary results. In this section we derive preliminary bounds and relations for several operators associated with the preconditioners $(6)-(7)$. We will assume that the discrete problem (3) arises from a standard finite difference or loworder finite element scheme on a uniform grid with mesh size $h$, and that the discrete problem is scaled so that the extreme eigenvalues of the discrete Laplace operators of $A$ are contained in an interval of the form $\left[c_{1} h^{2}, c_{2}\right]$, where here and below $c_{i}$ denotes a generic constant that is independent of $h$ and $\nu$. This is a natural scaling for finite elements, and for finite differences on a uniform grid it corresponds to the five-point operator with 4 in the diagonal entries and -1 in the off-diagonal entries. With this normalization, $B B^{T} / h^{2}$ is also a scaled discrete Laplace operator and its eigenvalues are contained in an interval of the same form. Let the discrete velocity and pressure spaces have dimension $n_{u}$ and $n_{p}$, respectively. For div-stable discretizations, $n_{u} \geq n_{p}$, and typically $n_{u}$ is significantly larger than $n_{p} .^{2}$

It will be convenient to use the symbol $Q$ instead of $\frac{1}{\nu} M$ in the matrices of (6). The preconditioned matrices are then given by

$$
\mathcal{A}_{D}=\left(\begin{array}{cc}
F & B^{T} \\
B & 0
\end{array}\right)\left(\begin{array}{cc}
F^{-1} & 0 \\
0 & Q^{-1}
\end{array}\right)=\left(\begin{array}{cc}
I & K^{T} \\
G & 0
\end{array}\right)
$$

for the block diagonal preconditioner, and

$$
\mathcal{A}_{T}=\left(\begin{array}{cc}
F & B^{T} \\
B & 0
\end{array}\right)\left(\begin{array}{cc}
F^{-1} & F^{-1} B^{T} Q^{-1} \\
0 & -Q^{-1}
\end{array}\right)=\left(\begin{array}{cc}
I & 0 \\
G & H
\end{array}\right)
$$

\footnotetext{
${ }^{2}$ For two-dimensional problems, the vector $\mathbf{u}$ has two components of grid vectors, and stability considerations often also lead to more grid points for velocity than for pressure [8].
} 
for the block triangular preconditioner. The submatrices on the right of $(8)-(9)$ are

$$
G=B F^{-1}, \quad K^{T}=B^{T} Q^{-1}, \quad H=G K^{T}=B F^{-1} B^{T} Q^{-1} .
$$

The identity matrices are of order $n_{u}$.

We recall some results from [3], which give bounds on the eigenvalues of $H$. Let $S=B F^{-1} B^{T}$ denote the Schur complement matrix for (3), and let

$$
C=B\left(\frac{F^{-1}+F^{-T}}{2}\right) B^{T}, \quad R=B\left(\frac{F^{-1}-F^{-T}}{2}\right) B^{T},
$$

denote the symmetric and skew-symmetric parts of $S$, respectively. It is shown in [3] that

$$
\frac{\gamma^{2} \nu^{2}}{\delta^{2}+\nu^{2}} \leq \frac{(q, C q)}{(q, Q q)} \leq \Gamma^{2}, \quad \frac{|(q, R q)|}{(q, Q q)} \leq \frac{\Gamma^{2}}{2}
$$

where $\gamma$ and $\Gamma$ are as in (5) and $\delta$ is the largest eigenvalue of $A^{-1} N$, which is also uniformly bounded independent of $h$ [4]. Consequently, Bendixson's theorem [14, p. $418]$ implies that the eigenvalues of $H$ are contained in the box

$$
\left[\frac{\gamma^{2} \nu^{2}}{\delta^{2}+\nu^{2}}, \Gamma^{2}\right] \times\left[\frac{-\Gamma^{2}}{2}, \frac{\Gamma^{2}}{2}\right]
$$

in the complex plane.

We first derive bounds on the singular values of $G$, which will be used in the perturbation analysisis for the block triangular preconditioner.

THEOREM 2.1. The largest singular value of $G$ is bounded above by a quantity of magnitude $O(1 / \nu)$ which is independent of $h$ as $h \rightarrow 0$. The smallest singular value of $G$ is bounded below by a quantify of magnitude $O(h)$ which is bounded independent of $\nu$ as $\nu \rightarrow 0$.

Proof. The singular values of $G$ are the square roots of the eigenvalues of $G G^{T}$, and the largest and smallest of these eigenvalues are the extrema of $\left(q, G G^{T} q\right) /(q, q)$. This Rayleigh quotient can be rewritten as

$$
\frac{\left(q, G G^{T} q\right)}{(q, q)}=\frac{\left(F^{-T} B^{T} q, F^{-T} B^{T} q\right)}{(q, q)}=\frac{\left(F^{-T} B^{T} q, F^{-T} B^{T} q\right)}{\left(F^{-T} B^{T} q, B^{T} q\right)} \frac{\left(F^{-T} B^{T} q, B^{T} q\right)}{(q, q)}
$$

We consider the two terms in the product on the right of (12) separately. For the first term, the substitution $w=F^{-T} B^{T} q$ gives

$$
\frac{\left(F^{-T} B^{T} q, F^{-T} B^{T} q\right)}{\left(F^{-T} B^{T} q, B^{T} q\right)}=\frac{(w, w)}{\left(w, F^{T} w\right)}=\frac{(w, w)}{\left(w,\left(\frac{F+F^{T}}{2}\right) w\right)}=\frac{1}{\nu} \frac{(w, w)}{(w, A w)} .
$$

Under the assumption on the scaling of the discrete Laplacian operators composing $A$, it follows that

$$
\frac{c_{1}}{\nu} \leq \frac{\left(F^{-T} B^{T} q, F^{-T} B^{T} q\right)}{\left(F^{-T} B^{T} q, B^{T} q\right)} \leq \frac{c_{2} h^{-2}}{\nu}
$$


The second term of the product in (12) is

$$
\frac{\left(q, B F^{-1} B^{T} q\right)}{(q, q)}=\frac{(q, C q)}{(q, q)}=\frac{(q, C q)}{(q, Q q)} \frac{(q, Q q)}{(q, q)} .
$$

It is well known (see [17]) that the pressure mass matrix is spectrally equivalent to $h^{2} I$, so that

$$
\frac{c_{1} h^{2}}{\nu} \leq \frac{(q, Q q)}{(q, q)} \leq \frac{c_{2} h^{2}}{\nu}
$$

Thus, the bounds for the symmetric part in (10) together with (12) - (15) imply

$$
\frac{c_{1} h^{2}}{\delta^{2}+\nu^{2}} \leq \frac{\left(q, G G^{T} q\right)}{(q, q)} \leq \frac{c_{2}}{\nu^{2}}
$$

口

The singular values of $K$ will be used to analyze the block diagonal preconditioner.

Lemma 2.2. The largest singular value of $K$ is bounded above by a quantity of magnitude $O\left(\frac{\nu}{h}\right)$. The smallest singular value is bounded below by a quantity of magnitude $O(\nu)$.

Proof. The largest singular value is $\|K\|_{2}=\left\|K^{T}\right\|_{2}$. Using $K^{T}=B^{T} Q^{-1}$ and $Q=\frac{1}{\nu} M$, we have

$$
\left\|K^{T}\right\|_{2} \leq \nu\left\|B^{T}\right\|_{2}\left\|M^{-1}\right\|_{2}
$$

But $\left\|M^{-1}\right\|_{2}=O\left(h^{-2}\right)$, and our assumptions on $B$ imply that $\left\|B^{T}\right\|_{2}=\left\|B B^{T}\right\|_{2}^{1 / 2}=$ $O(h)$. The smallest singular value is the inverse of $\left\|\left(K K^{T}\right)^{-1}\right\|_{2}^{1 / 2}$. Then

$$
\left\|\left(K K^{T}\right)^{-1}\right\|_{2} \leq \frac{1}{\nu^{2}}\|M\|_{2}^{2}\left\|\left(B B^{T}\right)^{-1}\right\|_{2}=\frac{1}{\nu^{2}} c_{1} h^{4} c_{2} h^{-4}
$$

Consider an alternative scaling in problems (1) and (4) in which the first equation is multiplied by $\frac{1}{\nu}$. This does not change the solutions, but as we will show in $\$ \S 3-4$, it affects the sensitivity of discrete eigenvalues. For (4), scaling gives

$$
-\Delta \mathbf{u}+\frac{1}{\nu}(\mathbf{w} \cdot \operatorname{grad}) \mathbf{u}+\operatorname{grad}\left(\frac{1}{\nu} p\right)=\frac{1}{\nu} \mathbf{f} .
$$

The new discrete problem is as in (3) except that $F, p$ and $\mathbf{f}$ are replaced by $\hat{F}=\frac{1}{\nu} F$, $\frac{1}{\nu} p$ and $\frac{1}{\nu}$ f, respectively. Let $\hat{Q}=\nu Q=M, \hat{G}=B \hat{F}^{-1}, \hat{K}^{T}=B^{T} \hat{Q}^{-1}$, and $\hat{H}=\hat{G} \hat{K}^{T}$. The analogues of (11) and the bounds of Theorem 2.1 and Lemma 2.2 are given below. The proof follows from the facts that $\hat{G}=\nu G, \hat{K}=\frac{1}{\nu} K$ and $\hat{H}=H$.

COROLLARY 2.3. With the scaling of (16), the eigenvalues of $\hat{H}$ are contained in the box (11); the singular values of $\hat{G}$ are bounded above by a quantity of magnitude $O(1)$ and below by a quantity of magnitude $O(h \nu)$; and the singular values of $\hat{K}^{r}$ are bounded above by a quantity of magnitude $O\left(\frac{1}{h}\right)$ and below by a quantity of magnitude $O(1)$. 
In the following, we will not specifically identify the matrices associated with this scaling using the "hat"-symbol. Instead, we will use the notation of (8) - (9) to refer generically to both scalings. We will distinguish them as derived from either the "original" formulation (4) or the "scaled" formulation (16) of the Oseen equations.

Finally, we will use the notation $\alpha_{G, K}$ to denote the secant of the largest principal angle between Range $\left(G^{T}\right)$ and Range $\left(K^{T}\right)$. That is, if $Q_{G}$ and $Q_{K}$ are matrices whose columns represent orthogonal bases of $\operatorname{Range}\left(G^{T}\right)$ and $\operatorname{Range}\left(K^{T}\right)$, respectively, then

$$
\alpha_{G, K}=\left\|\left(Q_{G}^{T} Q_{K}\right)^{-1}\right\|_{2}=\frac{1}{\sigma_{\min }\left(Q_{G}^{T} Q_{K}\right)},
$$

where $\sigma_{\min }$ denotes the smallest singular value (see [7, p. 584]). It is easily shown (e.g., using $Q R$ decompositions) that

$$
\alpha_{G, K}=\left\|K^{T}\left(G K^{T}\right)^{-1} G\right\|_{2} .
$$

3. Analytic bounds for the block triangular preconditioner. It is evident from (9) that the eigenvalues of $\mathcal{A}_{T}$ consist of $\lambda=1$ of multiplicity $n_{u}$ together with the eigenvalues of $H$. We seek a factorization

$$
\mathcal{A}_{T}=\mathcal{V}_{T} \mathcal{D}_{T} \mathcal{V}_{T}^{-1}
$$

that provides insight into the sensitivity of these eigenvalues to perturbation. We will look for factors of the form

$$
\mathcal{D}_{T}=\left(\begin{array}{cc}
I & 0 \\
D_{21} & \Lambda
\end{array}\right) \begin{gathered}
n_{u} \\
n_{p}
\end{gathered}, \quad \mathcal{V}_{T}=\left(\begin{array}{cc}
V_{11} & V_{12} \\
V_{21} & V_{22} \\
n_{u} & n_{p}
\end{array}\right) n_{p}
$$

where the sub-blocks of $\mathcal{D}_{T}$ and $\mathcal{V}_{T}$ must be determined and the dimensions are as indicated. Let $H$ have Jordan canonical form $H=P \Lambda P^{-1}$. The requirement $\mathcal{A}_{T} \mathcal{V}_{T}=$ $\mathcal{V}_{T} \mathcal{D}_{T}$ is satisfied if

$$
\begin{array}{ll}
V_{11}=I, & V_{12}=0 \\
G=(I-H) V_{21}+P D_{21}, & V_{22}=P .
\end{array}
$$

We distinguish between two cases, $1 \in \sigma(H)$ and $1 \notin \sigma(H)$.

Suppose first that $H$ has no eigenvalues equal to 1 . The choice $D_{21}=0$ in $(20)$ leads to $V_{21}=(I-H)^{-1} G$. In this case, (19) represents a Jordan form for $\mathcal{A}_{T}$ with

$$
\mathcal{V}_{T}=\left(\begin{array}{cc}
I & 0 \\
(I-H)^{-1} G & P
\end{array}\right), \quad \mathcal{V}_{T}^{-1}=\left(\begin{array}{cc}
I & 0 \\
-P^{-1}(I-H)^{-1} G & P^{-1}
\end{array}\right) .
$$

Let $\mathcal{A}_{T}(\epsilon)=\mathcal{A}_{T}+\epsilon \mathcal{E}$ be a perturbation of $\mathcal{A}_{T}$, where

$$
\mathcal{E}=\left(\begin{array}{ll}
E_{11} & E_{12} \\
E_{21} & E_{22}
\end{array}\right)
$$

The classical perturbation analysis of Wilkinson based on Gerschgorin theory [18, pp. 71ff] shows that for every eigenvalue $\lambda$ of $\mathcal{A}_{T}$ with only linear elementary divisors, perturbations of $\lambda$ in $\sigma\left(\mathcal{A}_{T}(\epsilon)\right)$ are contained in a circle centered at $\lambda$ with radius of 
size $c \epsilon$ where $c$ is independent of $\epsilon$. The structure of $\mathcal{V}_{T}$ can be used to obtain further insight into the sizes of the perturbations. Let

$$
\hat{\mathcal{E}}_{T}=\mathcal{V}_{T}^{-1} \mathcal{E} \mathcal{V}_{T}=\left(\begin{array}{cc}
\hat{E}_{11} & \hat{E}_{12} \\
\hat{E}_{21} & \hat{E}_{22}
\end{array}\right)
$$

so that we are concerned with the eigenvalues of

$$
\mathcal{V}_{T}^{-1} \mathcal{A}_{T}(\epsilon) \mathcal{V}_{T}=\mathcal{D}_{T}+\epsilon \hat{\mathcal{E}}_{T}=\left(\begin{array}{cc}
I & 0 \\
0 & \Lambda
\end{array}\right)+\epsilon\left(\begin{array}{cc}
\hat{E}_{11} & \hat{E}_{12} \\
\hat{E}_{21} & \hat{E}_{22}
\end{array}\right)
$$

Here and in the following, the symbol " $c$ " represents a generic constant that is independent of the parameters $h, \nu$ and $\epsilon$.

Lemma 3.1. If $1 \notin \sigma(H)$, then there are $n_{u}$ eigenvalues $\hat{\lambda}$ of $\mathcal{A}_{T}(\epsilon)$ (counting multiplicity) satisfying

$$
|\hat{\lambda}-1| \leq \epsilon\left\|\hat{E}_{11}\right\|_{\infty}+c \epsilon^{2}\left\|\hat{E}_{12}\right\|_{\infty}
$$

If there are $m \leq n_{p}$ eigenvalues $\lambda$ of $H$ with linear elementary divisors, then there are $m$ eigenvalues $\hat{\lambda}$ of $\mathcal{D}_{T}(\epsilon)$, distinct from those of (26), that satisfy

$$
|\hat{\lambda}-\lambda| \leq \epsilon\left\|\hat{E}_{22}\right\|_{\infty}+c \epsilon^{2}\left\|\hat{E}_{21}\right\|_{\infty} .
$$

Proof. Multiplying the first block row on the right side of (25) by $\epsilon / \beta$, multiplying the first block column by $\beta / \epsilon$ and then applying Gerschgorin's theorem leads to the bound

$$
\left|\hat{\lambda}-\left(1+\epsilon\left[\hat{E}_{11}\right]_{i i}\right)\right| \leq \epsilon \sum_{j \neq i}\left|\left[\hat{E}_{11}\right]_{i j}\right|+\epsilon^{2} / \beta \sum_{j}\left|\left[\hat{E}_{12}\right]_{i j}\right|,
$$

where $\beta$ is such that the Gerschgorin disks for the first and second blocks of the scaled matrix are disjoint (see [18, p. 73]). Assertion (26) follows. The argument for (27) is identical, applied to the second block row of (25).

Thus, we can restrict our attention to the block diagonal entries of $\hat{\mathcal{E}}_{T}$. Using (22) $-(24)$ we have

$$
\begin{aligned}
& \hat{E}_{11}=E_{11}+E_{12}(I-H)^{-1} G, \\
& \hat{E}_{22}=-P^{-1}(I-H)^{-1} G E_{12} P+P^{-1} E_{22} P .
\end{aligned}
$$

The following result gives bounds on the perturbations of eigenvalues as functions of the viscosity $\nu$ and mesh size $h$, for a perturbation satisfying $\|\mathcal{E}\|_{2} \leq 1$. $^{3}$

Theorem 3.2. Assume $\|\mathcal{E}\|_{2} \leq 1$. If $1 \notin \sigma(H)$, then the eigenvalues $\hat{\lambda}$ of $\mathcal{A}_{T}(\epsilon)$ that are perturbations of $\lambda=1$ satisfy

$$
|\hat{\lambda}-1| \leq\left\{\begin{array}{cl}
\epsilon \frac{c_{1}}{\nu h}+O\left(\epsilon^{2}\right) & \text { for the original formulation, } \\
\epsilon \frac{c_{1}}{h}+O\left(\epsilon^{2}\right) & \text { for the scaled formulation, }
\end{array}\right.
$$

\footnotetext{
${ }^{3}$ This assumption is stronger than the inequality $\left|\mathcal{E}_{i j}\right| \leq 1$ used by Wilkinson; the latter condition follows from our assumption.
} 
where $c_{1}=c\left\|(I-H)^{-1}\right\|_{2}$. For eigenvalues $\lambda$ of $H$ with linear elementary divisors, the perturbations $\hat{\lambda} \in \sigma\left(\mathcal{A}_{T}(\epsilon)\right)$ satisfy

$$
|\hat{\lambda}-\lambda| \leq\left\{\begin{array}{cl}
\epsilon \frac{c_{2}}{\nu h}+O\left(\epsilon^{2}\right) & \text { for the original formulation } \\
\epsilon \frac{c_{2}}{h}+O\left(\epsilon^{2}\right) & \text { for the scaled formulation }
\end{array}\right.
$$

where $c_{2}=c \kappa(P)\left\|(I-H)^{-1}\right\|_{2}$.

Proof. The relations $(28)$ together with standard bounds on matrix $l_{p}$-norms give

$$
\begin{aligned}
\left\|\hat{E}_{11}\right\|_{\infty} & \leq \frac{c}{h}\left\|\hat{E}_{11}\right\|_{2} \leq \frac{c}{h}\left(1+\left\|(I-H)^{-1}\right\|_{2}\|G\|_{2}\right), \\
\left\|\hat{E}_{22}\right\|_{\infty} & \leq \frac{c}{h}\left\|\hat{E}_{22}\right\|_{2} \leq \frac{c}{h} \kappa(P)\left(\left\|(I-H)^{-1}\right\|_{2}\|G\|_{2}+1\right) .
\end{aligned}
$$

The conclusions follow from the upper bounds on $\|G\|_{2}$ in Theorem 2.1. $\square$

In the case $1 \in \sigma(H)$, we use an alternative version of (20). By analogy with the analysis above, let $V_{21}=(I-H)^{\dagger} G$ where $(I-H)^{\dagger}$ is the pseudo-inverse. Then

$$
\mathcal{V}_{T}=\left(\begin{array}{cc}
I & 0 \\
(I-H)^{\dagger} G & P
\end{array}\right), \quad \mathcal{V}_{T}^{-1}=\left(\begin{array}{cc}
I & 0 \\
-P^{-1}(I-H)^{\dagger} G & P^{-1}
\end{array}\right)
$$

The similarity (19) then holds for the choice

$$
\mathcal{D}_{T}=\left(\begin{array}{cc}
I & 0 \\
P^{-1} \Pi G & \Lambda
\end{array}\right)
$$

where $\Pi$ is the orthogonal projection onto the null space of $I-H^{T}$. The perturbation of $\mathcal{D}_{T}$ associated with $\mathcal{A}_{T}(\epsilon)$ is then

$$
\mathcal{V}_{T}^{-1} \mathcal{A}_{T}(\epsilon) \mathcal{V}_{T}=\mathcal{D}_{T}+\epsilon \hat{\mathcal{E}}_{T}=\left(\begin{array}{cc}
I & 0 \\
0 & \Lambda
\end{array}\right)+\left(\begin{array}{cc}
\epsilon \hat{E}_{11} & \epsilon \hat{E}_{12} \\
-P^{-1} \Pi G+\epsilon \hat{E}_{21} & \epsilon \hat{E}_{22}
\end{array}\right)
$$

First consider the eigenvalues of $H$ different from 1 with linear elementary divisors. Let $\eta$ be a parameter in $(0,1)$. Multiplying the second block row of (31) by $\epsilon^{\eta}$ and the second block column by $\epsilon^{-\eta}$ produces the matrix

$$
\left(\begin{array}{cc}
I & 0 \\
0 & \Lambda
\end{array}\right)+\left(\begin{array}{cc}
\epsilon \hat{E}_{11} & \epsilon^{1-\eta} \hat{E}_{12} \\
-\epsilon^{\eta} P^{-1} \Pi G+\epsilon^{1+\eta} \hat{E}_{21} & \epsilon \hat{E}_{22}
\end{array}\right)
$$

For any $\eta \in(0,1)$ and small enough $\epsilon$, the Gerschgorin disk for a perturbation $\hat{\lambda}$ of $\lambda \in \sigma(H), \lambda \neq 1$, is disjoint from the disks corresponding to perturbations of the eigenvalue 1 . Consequently, if $\lambda$ has only linear elementary divisors, then

$$
|\hat{\lambda}-\lambda| \leq \epsilon^{\eta}\left\|P^{-1} \Pi G\right\|_{\infty}+O(\epsilon)
$$

For eigenvalues equal to 1 , multiplying the second block row of (31) by $\epsilon^{1 / 2}$ and multiplying the second block column by $\epsilon^{-1 / 2}$ produces

$$
\left(\begin{array}{cc}
I & 0 \\
0 & \Lambda
\end{array}\right)+\left(\begin{array}{cc}
\epsilon \hat{E}_{11} & \epsilon^{1 / 2} \hat{E}_{12} \\
-\epsilon^{1 / 2} P^{-1} \Pi G+\epsilon^{3 / 2} \hat{E}_{21} & \epsilon \hat{E}_{22}
\end{array}\right)
$$


Thus, for $\lambda=1$ with linear or quadratic elementary divisors, the perturbations satisfy

$$
|\hat{\lambda}-\lambda| \leq \epsilon^{1 / 2} \max \left(\left\|\hat{E}_{12}\right\|_{\infty},\left\|P^{-1} \Pi G\right\|_{\infty}\right)+O(\epsilon) .
$$

Bounding the matrix infinity norms in (32) and (33) gives the following result.

THEOREM 3.3. If $1 \in \sigma(H)$ with linear or quadratic elementary divisors, then the eigenvalues $\hat{\lambda}$ of $\mathcal{A}_{T}(\epsilon)$ that are perturbations of $\lambda=1$ satisfy

$$
|\hat{\lambda}-1| \leq \epsilon^{1 / 2} \frac{c}{h} \max \left(\|P\|_{2},\left\|P^{-1}\right\|_{2}\|G\|_{2}\right)+O(\epsilon)
$$

For $\lambda \in \sigma(H)$ different from 1 with linear elementary divisors, the perturbations $\hat{\lambda} \in \sigma\left(\mathcal{A}_{T}(\epsilon)\right)$ satisfy

$$
|\hat{\lambda}-\lambda| \leq \epsilon^{\eta} \frac{c}{h}\left\|P^{-1}\right\|_{2}\|G\|_{2}+O(\epsilon)
$$

for any $\eta \in(0,1)$. Here $\|G\|_{2}=O(1 / \nu)$ in the original formulation and $\|G\|_{2}=O(1)$ for the scaled formulation.

Note that for any $\lambda \in \sigma\left(\mathcal{A}_{T}\right)$ with nonlinear elementary divisors, bounds analogous to $(29)-(30)$ and $(34)-(35)$ can be obtained in which the dependence on $\epsilon$ is of the form $\epsilon^{1 / r}$ where $r$ is the order of the largest Jordan block for $\lambda$; see [18, p. 79].

4. Analytic bounds for the block diagonal preconditioner. For the block diagonal preconditioner, we have results only in the case where the Schur complement matrix $H$ has no nonlinear elementary divisors. As in Theorem 3.2, the perturbation analysis will be stated in terms of properties of $P$, a fixed matrix of eigenvectors of $H$. In addition, some of the bounds depend on $\alpha_{G, K}$, the secant of the largest principal angle between Range $\left(G^{T}\right)$ and Range $\left(K^{T}\right)$, as defined in (17).

Consider the eigenvalue problem for $\mathcal{A}_{D}$,

$$
\left(\begin{array}{cc}
I & K^{T} \\
G & 0
\end{array}\right)\left(\begin{array}{l}
u \\
p
\end{array}\right)=\theta\left(\begin{array}{l}
u \\
p
\end{array}\right) .
$$

This leads to the conditions

$$
K^{T} p=(\theta-1) u, \quad G u=\theta p
$$

on the components of the eigenvectors. One solution corresponds to the eigenvalue $\theta=1$; the assumption that $B$ and therefore $K$ have full rank then implies that any associated eigenvector satisfies $p=0, G u=0$. We use two approaches to identify eigenvalues $\theta \neq 1$ and corresponding eigenvectors.

1. Substitution of $u=\left(\frac{1}{\theta-1}\right) K^{T} p$ into the second equation of (36) gives

$$
G K^{T} p=\theta(\theta-1) p .
$$

That is, any eigenpair $(\lambda, p)$ of $H=G K^{T}$ leads to two eigenvalues

$$
\theta_{+}=\frac{1+\sqrt{1+4 \lambda}}{2}, \quad \theta_{-}=\frac{1-\sqrt{1+4 \lambda}}{2}
$$

of $\mathcal{A}_{D}$. The associated eigenvectors are

$$
\left(\begin{array}{c}
u_{+} \\
p
\end{array}\right), \quad\left(\begin{array}{c}
u_{-} \\
p
\end{array}\right),
$$


where

$$
u_{+}=\left(\frac{1}{\theta_{+}-1}\right) K^{T} p, \quad u_{-}=\left(\frac{1}{\theta_{-}-1}\right) K^{T} p .
$$

2. Alternatively, substitution of $p=\frac{1}{\theta} G u$ into the first equation of (36) gives

$$
K^{T} G u=\theta(\theta-1) u .
$$

$K^{T} G$ has a zero eigenvalue of multiplicity $n_{u}-n_{p}$ (the dimension of the null space of $G$ ), plus $2 n_{p}$ nonzero eigenvalues. If $(\lambda, u)$ is an eigenpair with $\lambda \neq 0$, then (37) defines a pair of eigenvalues of $\mathcal{A}_{D}$. In this case, the eigenvectors have the form

$$
\left(\begin{array}{c}
u \\
p_{+}
\end{array}\right), \quad\left(\begin{array}{c}
u \\
p_{-}
\end{array}\right),
$$

where

$$
p_{+}=\left(\frac{1}{\theta_{+}}\right) G u, \quad p_{-}=\left(\frac{1}{\theta_{-}}\right) G u .
$$

It is straightforward to show that any $u_{+}$or $u_{-}$from $(39)$ is an eigenvector of $K^{T} G$, and any $p_{+}$or $p_{-}$from (41) is an eigenvector of $G K^{T}$. Therefore, we will use the symbol " \pm " to refer to the pairs of eigenvalues and eigenvectors of $\mathcal{A}_{D}$, i.e., $u_{ \pm}$ will refer to the first entries of either (38) or (40), $p_{ \pm}$to the second entries, and $\theta_{ \pm}$to the associated eigenvalue. We have established the following result.

THEOREM 4.1. The eigenvectors of $\mathcal{A}_{D}$ corresponding to the eigenvalue $\theta=1$ have the form $\left(\begin{array}{l}u \\ 0\end{array}\right)$ where $G u=0$. The eigenvectors corresponding to eigenvalues different from 1 have the form $\left(\begin{array}{c}u_{ \pm} \\ p_{ \pm}\end{array}\right)$, where the associated eigenvalues $\theta_{ \pm}$satisfy (37), and

$$
H p_{ \pm}=\lambda p_{ \pm}, \quad K^{T} G u_{ \pm}=\lambda u_{ \pm}, \quad p_{ \pm}=\left(\frac{1}{\theta_{ \pm}}\right) G u_{ \pm}, \quad u_{ \pm}=\left(\frac{1}{\theta_{ \pm}-1}\right) K^{T} p_{ \pm} .
$$

Let $\Theta_{ \pm}$denote a diagonal matrix with entries $\left\{\theta_{ \pm}\right\}$from (37). If the eigenvalues of $H$ have only linear elementary divisors, then $\mathcal{A}_{D} \mathcal{V}_{D}=\mathcal{V}_{D} \mathcal{D}_{D}$, where

$$
\mathcal{D}_{D}=\left(\begin{array}{ccc}
I & 0 & 0 \\
0 & \Theta_{+} & 0 \\
0 & 0 & \Theta_{-}
\end{array}\right) \begin{aligned}
& n_{u}-n_{p} \\
& n_{p} \\
& n_{p} \\
& n_{u}- \\
& n_{p}
\end{aligned} n_{p} n_{p} \quad, \quad \mathcal{V}_{D}=\left(\begin{array}{c}
V U_{+} U_{-} \\
0 P_{+} P_{-}
\end{array}\right) n_{p} n_{u},
$$

in which the columns of $V$ form an orthogonal basis of the null space of $G$ and

$$
P_{ \pm}=G U_{ \pm} \Theta_{ \pm}^{-1}, \quad U_{ \pm}=K^{T} P_{ \pm}\left(\Theta_{ \pm}-I\right)^{-1} .
$$

We will normalize the matrices $U_{ \pm}$and $P_{ \pm}$as follows:

$$
\begin{aligned}
& U_{+}=U_{-}=U=K^{T} P, \\
& P_{+}=P\left(\Theta_{+}-I\right), \\
& P_{-}=P\left(\Theta_{-}-I\right) .
\end{aligned}
$$


It is then easily verified that

$$
\mathcal{V}_{D}^{-1}=\left(\begin{array}{ccc}
I & 0 & 0 \\
0 & \left(\Theta_{+}-\Theta_{-}\right)^{-1} & 0 \\
0 & 0 & \left(\Theta_{-}-\Theta_{+}\right)^{-1}
\end{array}\right)\left(\begin{array}{cc}
\Phi & 0 \\
P_{+}^{-1} G & P^{-1} \\
P_{-}^{-1} G & P^{-1}
\end{array}\right)
$$

where

$$
\Phi=V^{T}\left(I-U(G U)^{-1} G\right)=V^{T}\left(I-K^{T}\left(G K^{T}\right)^{-1} G\right) .
$$

Now let $\mathcal{A}_{D}(\epsilon)=\mathcal{A}_{D}+\epsilon \mathcal{E}$ be a perturbation of $\mathcal{A}_{D}$ where $\mathcal{E}$ is as in (23). The eigenvalues of this perturbed matrix are the same as those of

$$
\mathcal{V}_{D}^{-1} \mathcal{A}_{D}(\epsilon) \mathcal{V}_{D}=\mathcal{D}_{D}+\epsilon \hat{\mathcal{E}}_{D}=\left(\begin{array}{ccc}
I+\epsilon \hat{E}_{11} & \epsilon \hat{E}_{12} & \epsilon \hat{E}_{13} \\
\epsilon \hat{E}_{21} & \Theta_{+}+\epsilon \hat{E}_{22} & \epsilon \hat{E}_{23} \\
\epsilon \hat{E}_{31} & \epsilon \hat{E}_{32} & \Theta_{-}+\epsilon \hat{E}_{33}
\end{array}\right),
$$

where $\hat{\mathcal{E}}_{D}=\mathcal{V}_{D}^{-1} \mathcal{E} \mathcal{V}_{D}$. As in $\S 3$, Gerschgorin analysis implies that for small $\epsilon$, the effects of perturbation can be bounded using the perturbations on the block diagonal of (45). These are given by

$$
\begin{aligned}
& \hat{E}_{11}=\Phi^{T} E_{11} V, \\
& \hat{E}_{22}=\left(\Theta_{+}-\Theta_{-}\right)^{-1}\left[P_{+}^{-1} G\left(E_{11} U+E_{12} P_{+}\right)+P^{-1}\left(E_{21} U+E_{22} P_{+}\right)\right], \\
& \hat{E}_{33}=\left(\Theta_{-}-\Theta_{+}\right)^{-1}\left[P_{-}^{-1} G\left(E_{11} U+E_{12} P_{-}\right)+P^{-1}\left(E_{21} U+E_{22} P_{-}\right)\right] .
\end{aligned}
$$

THEOREM 4.2. If $H$ has no nonlinear elementary divisors, then the eigenvalues $\hat{\theta}$ of $\mathcal{A}_{D}(\epsilon)$ that are perturbations of $\theta=1$ satisfy

$$
|\hat{\theta}-1| \leq \epsilon \frac{c}{h}\left(1+\alpha_{G, K}\right)+O\left(\epsilon^{2}\right)
$$

for both the original and scaled formulations of the discrete Oseen equations. The perturbations of eigenvalues different from 1 satisfy

$$
\left|\hat{\theta}_{+}-\theta_{+}\right| \leq \begin{cases}\epsilon \kappa(P) \alpha_{G, K}\left(\frac{c_{1}}{h^{2}}+\frac{c_{2}}{\nu h}\right)+O\left(\epsilon^{2}\right) & \text { for the original formulation, } \\ \epsilon \kappa(P) \alpha_{G, K} \frac{c}{h^{2}}+O\left(\epsilon^{2}\right) & \text { for the scaled formulation, }\end{cases}
$$

and

$$
\left|\hat{\theta}_{-}-\theta_{-}\right| \leq \begin{cases}\epsilon \kappa(P)\left(\frac{c_{1}}{h^{2}}+\frac{c_{2}}{\nu h}\right)+O\left(\epsilon^{2}\right) & \text { for the original formulation, } \\ \epsilon \kappa(P) \frac{c}{h^{2}}+O\left(\epsilon^{2}\right) & \text { for the scaled formulation. }\end{cases}
$$

Proof. For the perturbations of $\lambda=1$, it follows from (18), (44) and (45) that

$$
|\hat{\theta}-1| \leq \epsilon c\left\|\hat{E}_{11}\right\|_{\infty} \leq \epsilon \frac{c}{h}\left\|\hat{E}_{11}\right\|_{2} \leq \epsilon \frac{c}{h}\left(1+\alpha_{G, K}\right) .
$$


For the perturbations of eigenvalues different from 1, note that (37) and the bounds (11) on the eigenvalues of $H$ imply that the norm of each of the diagonal matrices

$$
\begin{array}{lll}
\Theta_{+}-I, & \Theta_{-}-I, & \left(\Theta_{+}-\Theta_{-}\right)^{-1}, \\
\Theta_{+}^{-1}, & \left(\Theta_{-}-I\right)^{-1}, &
\end{array}
$$

is bounded independent of $\nu$ and $h$. Gerschgorin analysis gives

$$
\left|\hat{\theta}_{ \pm}-\theta_{ \pm}\right| \leq \epsilon \frac{c}{h}\left\|\hat{E}_{j j}\right\|_{2}
$$

where $j=2$ corresponds to $\Theta_{+}$and $j=3$ to $\Theta_{-}$. Relations (43) and (46) then imply

$$
\left\|\hat{E}_{j j}\right\|_{2} \leq\left\|\left(\Theta_{+}-\Theta_{-}\right)^{-1}\right\|_{2}\left(\left\|P_{ \pm}^{-1} G\right\|_{2}+\left\|P^{-1}\right\|_{2}\right)\left(\left\|K^{T}\right\|_{2}+\left\|\Theta_{ \pm}-I\right\|_{2}\right)\|P\|_{2}
$$

Consider $\left\|P_{-}^{-1} G\right\|_{2}$. Using the expression for $P_{-}$in (43), we have

$$
\left\|P_{-}^{-1} G\right\|_{2} \leq\left\|\left(\Theta_{-}-I\right)^{-1}\right\|_{2}\left\|P^{-1}\right\|_{2}\|G\|_{2},
$$

and combining this with (51) gives

$$
\left\|\hat{E}_{33}\right\|_{2} \leq \kappa(P)\left\|\left(\Theta_{-}-\Theta_{+}\right)^{-1}\right\|_{2}\left(\left\|\left(\Theta_{-}-I\right)^{-1}\right\|_{2}\|G\|_{2}+1\right)\left(\left\|K^{T}\right\|_{2}+\left\|\Theta_{-}-I\right\|_{2}\right) .
$$

Result (48) follows from (50), the boundedness of the matrices of (49), and the bounds on $\|G\|_{2}$ and $\left\|K^{T}\right\|_{2}$ in Lemma 2.2 and Corollary 2.3 .

For $\left\|P_{+}^{-1} G\right\|_{2}$, first observe that (42) and (43) imply

$$
\left(P_{+}^{-1} G\right)\left(K^{T} P \Theta_{+}^{-1}\right)=I .
$$

Let $\left(P_{+}^{-1} G\right)^{T}=Q_{1} R_{1}$ and $K^{T} P \Theta_{+}^{-1}=Q_{2} R_{2}$ be $Q R$ factorizations, where $R_{1}$ and $R_{2}$ are square and nonsingular. Then $R_{1}^{T} Q_{1}^{T} Q_{2} R_{2}=I$, and

$$
\left\|P_{+}^{-1} G\right\|_{2}=\left\|R_{1}\right\|_{2} \leq\left\|R_{2}^{-1}\right\|_{2}\left\|\left(Q_{1}^{T} Q_{2}\right)^{-1}\right\|_{2}=\alpha_{G, K}\left\|R_{2}^{-1}\right\|_{2} .
$$

But $\left\|R_{2}^{-1}\right\|_{2}$ is the inverse of the smallest singular value of $K^{T} P \Theta_{+}^{-1}$, so that

$$
\left\|R_{2}^{-1}\right\|_{2} \leq\left\|\Theta_{+} P^{-1}\left(K K^{T}\right)^{-1} P^{-T} \Theta_{+}\right\|_{2}^{1 / 2} \leq\left\|\Theta_{+}\right\|_{2}\left\|P^{-1}\right\|_{2}\left\|\left(K K^{T}\right)^{-1}\right\|_{2}^{1 / 2} .
$$

From Lemma 2.2, Corollary 2.3 and the boundedness of $\left\|\Theta_{+}\right\|_{2}$, the term on the right is bounded by $c / \nu\left\|P^{-1}\right\|_{2}$ for the original formulation and $c\left\|P^{-1}\right\|_{2}$ for the alternative formulation. Result (47) then follows from (50), (51) (with $j=2$ ) and (52).

REMARK 4.1. The difference between (47) and (48) stems from the fact that $\|\left(\Theta_{+}-\right.$ $I)^{-1} \|_{2}$ is not independent of $\nu$, so we cannot bound $\left\|P_{+}^{-1} G\right\|_{2}$ directly. The results of $\S 6$ suggest that the perturbations do not behave differently, but we see no way to avoid including $\alpha_{G, K}$ in (47).

5. The rank-deficient case. Unless an additional constraint is imposed on the pressure in (1)-(2) or (4), the matrix of (3) will be rank deficient by one. This is the case for the test problems of $\S 6$. We outline here how the analysis above carries over in the rank-deficient case. 
For the block triangular preconditioner, $\mathcal{A}_{T} \mathcal{V}_{T}=\mathcal{V}_{T} \mathcal{D}_{T}$ where, for $1 \notin \sigma(H)$, the analogues of the matrices defined in $(20)-(22)$ are

$$
\mathcal{D}_{T}=\left(\begin{array}{cc}
I & 0 \\
0 & \Lambda
\end{array}\right), \mathcal{V}_{T}=\left(\begin{array}{cc}
I & 0 \\
(I-H)^{-1} G & P
\end{array}\right), \mathcal{V}_{T}^{\dagger}=\left(\begin{array}{cc}
I & 0 \\
-P^{\dagger}(I-H)^{-1} G & P^{\dagger}
\end{array}\right)
$$

$\Lambda$ is a square matrix of order $n_{p}-1$ whose eigenvalues are the nonzero eigenvalues of $H$, and $P$ spans the associated invariant subspace. In particular, $P^{\dagger}$ replaces $P^{-1}$ and $\mathcal{V}_{T}^{\dagger}$ replaces $\mathcal{V}_{T}^{-1}$. The analysis of perturbations of the nonzero eigenvalues of $\mathcal{A}_{T}$ then carries through verbatim with the inverse of the smallest singular value of $P$ in place of $\left\|P^{-1}\right\|_{2}$. The case $1 \in \sigma(H)$ is generalized in a similar manner with $(I-H)^{\dagger}$ in place of $(I-H)^{-1}$

For the block diagonal preconditioner, the analogue of the similarity transformation of Theorem 4.1 is $\mathcal{A}_{D} \mathcal{V}_{D}=\mathcal{V}_{D} \mathcal{D}_{D}$ where

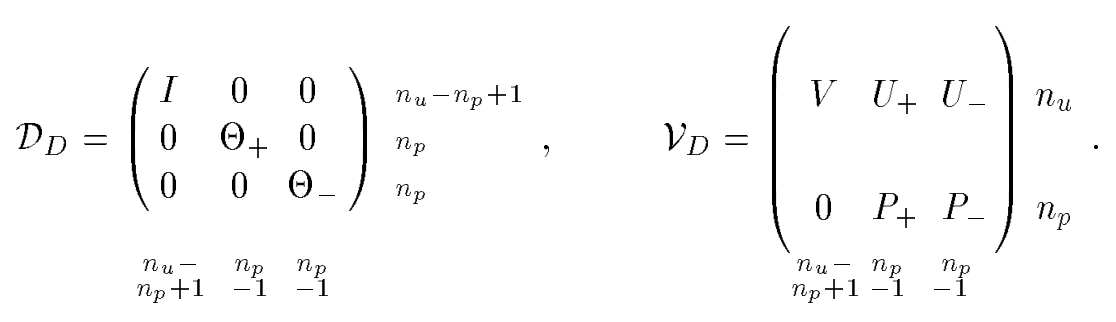

Once again, all the analysis of $\S 4$ goes through with $P$ referring to the matrix of eigenvectors corresponding to nonzero eigenvalues of $H, P^{\dagger}$ in place of $P^{-1}$, and the inverse of the smallest nonzero eigenvalue of $K K^{T}$ in place of $\left\|\left(K K^{T}\right)^{-1}\right\|_{2}$.

6. Experimental results. We now present the results of numerical experiments that supplement the analysis of $\$ \S 3-5$. Our test problem is a discrete Oseen operator (4) on on $\Omega=(-1,1) \times(-1,1)$, with Dirichlet boundary conditions $u_{1}=u_{2}=0$ on the three fixed walls $(x=-1, y=-1, x=1)$, and $u_{1}=1, u_{2}=0$ on the moving wall $(y=1)$. The coefficients of the convection terms describe a circular vortex

$$
w_{1}=2 y\left(1-x^{2}\right), \quad w_{2}=-2 x\left(1-y^{2}\right) .
$$

We discretize using bilinear finite elements on a uniform rectangular $n \times n$ velocity grids of width $h=2 / n$, augmented by streamline upwinding [9, p. 185]. To impose div-stability, the pressure discretization uses "macro-elements" of width $2 h$; see $[8$, p. 30]. The hydrostatic pressure is not explicitly specified, so that the matrices (3) are rank-deficient by one. Additional details about this problem are given in [3]. All computations were performed in MATLAB on either a Sun SPARC-20 workstation or a DEC-Alpha 2100 4/275 workstation.

We first show in Figure 1 some sample distributions of eigenvalues of $\mathcal{A}_{T}$ and $\mathcal{A}_{D}$, for $\nu=1 / 20$ and $n=32$. The plot on the left gives an indication of the rectangle enclosing the eigenvalues of $H$ (see (11)); the plot on the right represents the result of the mapping $\lambda \mapsto(1 \pm \sqrt{1+4 \lambda}) / 2$ of $(37)$. Both pictures include the eigenvalue 1 , of multiplicity $n_{u}=2178$ for $\mathcal{A}_{T}$ and $n_{u}-n_{p}+1=1890$ for $\mathcal{A}_{D}$.

We present our results primarily as tabulations of maximum perturbations and other quantities, for various choices of viscosity parameter $\nu$ and grid parameter $n$. The rows and columns of the tables indicate behavior as either $\nu \rightarrow 0$ or $n$ gets large $(h \rightarrow 0)$. Note that accurate discrete solutions to (4) are obtained only if $\nu$ is not too 
FIG. 1. Eigenvalues of $\mathcal{A}_{T}$ (left) and $\mathcal{A}_{D}$ (right), for $\nu=1 / 20$ and $n=32$.
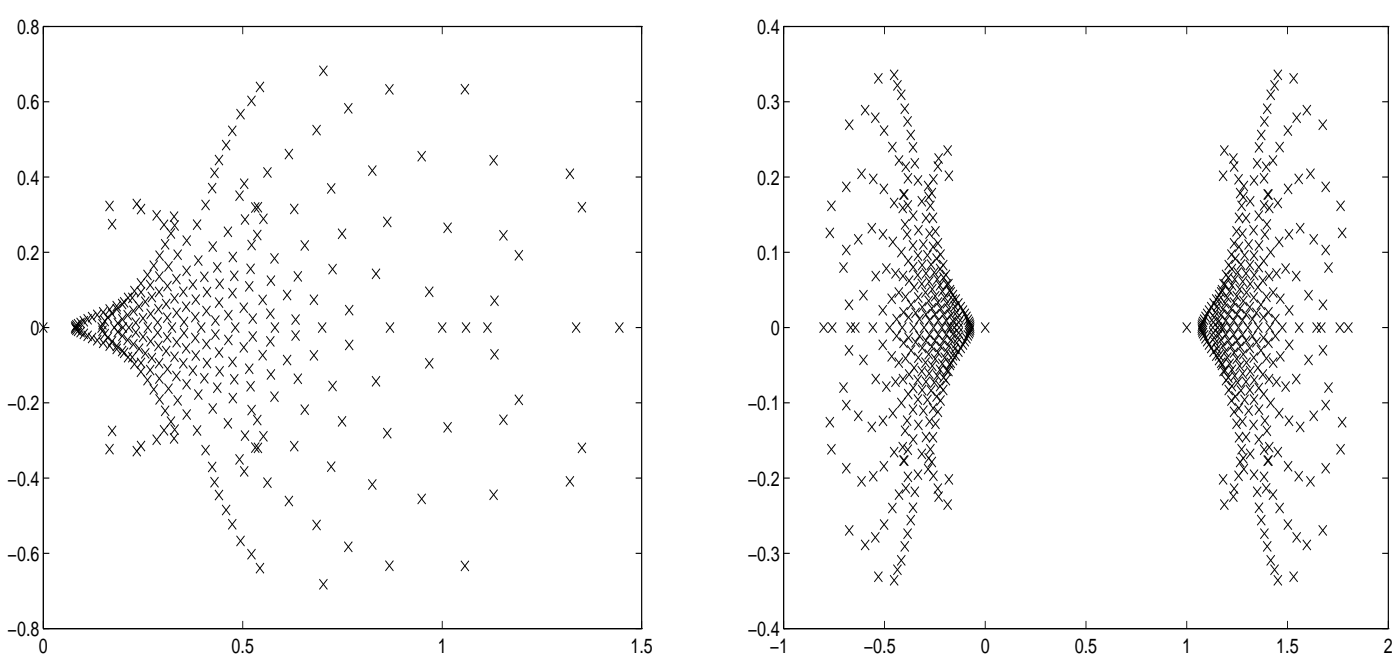

small relative to $h$. This difficulty can be ameliorated to some extent by an appropriate choice of discretization such as the streamline upwinding method used here [2],[9, p. 262]. In practical experiments, it is often desired to compute solutions of a fixed accuracy for a variety of values of $\nu$ by letting $h \rightarrow 0$ and $\nu \rightarrow 0$ simultaneously. In an effort to follow trends in the data, we will consider some combinations of $\nu$ and $h$ that could produce inaccurate solutions.

Our main results are in Tables 1 and 2. Table 1 shows the effects of perturbation of the block tridiagonal preconditioner, for two values of $n$ and various $\nu$. This data was obtained by computing the eigenvalues of a set of ten perturbed matrices $\mathcal{A}_{T}(\epsilon)=\mathcal{A}_{T}+\epsilon E$ where $\epsilon=10^{-8}$ and $E$ is a dense matrix with uniformly distributed random numbers in an interval $\left[-a_{n}, a_{n}\right]$, where $a_{n}=16 / n$. (For this choice, $\|E\|_{2}$ is approximately constant for all $n$.) For $\lambda=1$, the table presents

$$
\left(\max _{\hat{\lambda} \in \sigma_{1}\left(\mathcal{A}_{T}+\epsilon E\right)}|\hat{\lambda}-1|\right) / \epsilon,
$$

where $\sigma_{1}\left(\mathcal{A}_{T}+\epsilon E\right)$ is the set of $n_{p}$ eigenvalues that are closest to 1 , and the maximum is over all the perturbations. For $\lambda \neq 1$, the table shows

$$
\left(\max _{\hat{\lambda} \in \sigma_{1}^{\prime}\left(\mathcal{A}_{T}+\epsilon E\right)}|\hat{\lambda}-\lambda|\right) / \epsilon,
$$

where $\sigma_{1}^{\prime}\left(\mathcal{A}_{T}+\epsilon E\right)$ denotes the perturbations of $\lambda \in \sigma\left(\mathcal{A}_{T}\right), \lambda \neq 1$ and $\lambda \neq 0 .{ }^{4}$ Analogous results for the block diagonal preconditioner are shown in Table 2. Here we also distinguish between the eigenvalues with positive and negative real parts.

Before discussing this data, we present experimental results for three other quantities appearing in the bounds of $\S \S 3-4: \kappa(P),\left\|(I-H)^{-1}\right\|_{2}$, and $\alpha_{G, K}$. These are shown in Tables 3,4 , and 5 , respectively. Here $\kappa(P)$ refers to the version used for the

\footnotetext{
${ }^{4}$ These were obtained by sorting $\sigma\left(\mathcal{A}_{T}\right)$ and $\sigma\left(\mathcal{A}_{T}+E\right)$ for each $E$ and comparing the ordered sets. For all tests, similar results were obtained for other values of $\epsilon$.
} 
TABLE 1

Maximum normalized perturbations of eigenvalues of $\mathcal{A}_{T}$.

\begin{tabular}{c|c|cccccc}
$n=16$ & $\nu$ & 1 & $1 / 10$ & $1 / 20$ & $1 / 30$ & $1 / 50$ & $1 / 100$ \\
\hline Original & $\lambda=1$ & 21.7 & 137.6 & 318.5 & 478.2 & 766.1 & 1509.5 \\
Formulation & $\lambda \neq 1$ & 21.4 & 80.5 & 201.1 & 313.4 & 529.9 & 1110.0 \\
\hline Scaled & $\lambda=1$ & 21.7 & 16.7 & 16.9 & 16.9 & 16.9 & 17.1 \\
Formulation & $\lambda \neq 1$ & 21.4 & 8.3 & 10.2 & 10.6 & 10.7 & 11.2 \\
\hline & \multicolumn{7}{|c}{} \\
$n=32$ & $\lambda=1$ & 15.7 & 58.6 & 56.6 & 83.0 & 172.0 & 402.4 \\
\hline Original & $\lambda \neq 1$ & 22.7 & 26.7 & 50.8 & 43.1 & 141.2 & 257.8 \\
Formulation & $\lambda \neq 1$ & 15.7 & 15.2 & 14.0 & 14.1 & 14.2 & 14.4 \\
\hline Scaled & $\lambda=1$ & 11.1 & 93.0 & 35.7
\end{tabular}

TABLE 2

Maximum normalized perturbations of eigenvalues of $\mathcal{A}_{D}$.

\begin{tabular}{c|c|cccccc}
$n=16$ & $\nu$ & 1 & $1 / 10$ & $1 / 20$ & $1 / 30$ & $1 / 50$ & $1 / 100$ \\
\hline Original & $\lambda=1$ & 13.4 & 13.5 & 13.7 & 14.2 & 14.8 & 15.8 \\
Formulation & $\operatorname{Re}(\lambda)<0$ & 11.6 & 7.5 & 6.5 & 9.8 & 16.2 & 31.9 \\
& $\operatorname{Re}(\lambda)>1$ & 10.1 & 8.3 & 7.6 & 10.6 & 16.5 & 32.1 \\
\hline Scaled & $\lambda=1$ & 13.4 & 13.5 & 13.7 & 14.2 & 14.8 & 15.8 \\
Formulation & $\operatorname{Re}(\lambda)<0$ & 11.6 & 45.3 & 9.0 & 10.0 & 15.6 & 20.7 \\
& $\operatorname{Re}(\lambda)>1$ & 10.1 & 44.3 & 9.4 & 10.5 & 15.9 & 23.4 \\
\hline \multirow{3}{*}{$n=32$} & \multicolumn{7}{|c}{} \\
Original & $\lambda=1$ & 12.9 & 12.9 & 13.0 & 13.0 & 13.1 & 13.7 \\
Formulation & $\operatorname{Re}(\lambda)<0$ & 55.6 & 5.1 & 2.8 & 9.4 & 74.9 & 51.2 \\
& $\operatorname{Re}(\lambda)>1$ & 46.6 & 7.4 & 4.0 & 8.4 & 71.8 & 58.0 \\
\hline Scaled & $\lambda=1$ & 12.9 & 12.9 & 13.0 & 13.0 & 13.1 & 13.7 \\
Formulation & $\operatorname{Re}(\lambda)<0$ & 55.6 & 48.7 & 19.0 & 60.4 & 505.6 & 324.3 \\
& $\operatorname{Re}(\lambda)>1$ & 46.6 & 47.7 & 19.6 & 57.6 & 516.7 & 301.1
\end{tabular}


TABLE 3

Condition number of matrix $P$ of eigenvectors of $H$.

\begin{tabular}{c|cccccc}
$\nu$ & 1 & $1 / 10$ & $1 / 20$ & $1 / 30$ & $1 / 50$ & $1 / 100$ \\
\hline$n=16$ & 5.4 & 18.0 & 5.4 & 5.0 & 7.9 & 14.7 \\
$n=32$ & 60.8 & 45.7 & 26.8 & 78.5 & 557.0 & 413.3 \\
$n=64$ & 181.8 & 3876.0 & 2900.4 & 2029.1 & 5441.2 & $2.74 \mathrm{e} 4$
\end{tabular}

TABLE 4

Norm of $(I-H)^{-1}$.

\begin{tabular}{c|cccccc}
$\nu$ & 1 & $1 / 10$ & $1 / 20$ & $1 / 30$ & $1 / 50$ & $1 / 100$ \\
\hline$n=16$ & 100.8 & 16.2 & 17.1 & 17.1 & 16.5 & 16.6 \\
$n=32$ & 70.8 & 70.9 & 23.4 & 16.7 & 43.2 & 17.3 \\
$n=64$ & 697.0 & $2.59 \mathrm{e} 4$ & 1124.9 & 318.7 & 61.9 & 231.1
\end{tabular}

rank-deficient problem as outlined in $\S 5$. It was obtained by computing the matrix of eigenvectors $P$ of $H$, normalizing the columns of $P$ to have unit $l_{2}$-norm, and then computing the ratio of largest to smallest singular values of the submatrix of $P$ corresponding to the nonzero eigenvalues of $H$. (The normalization ensures that we are within a factor of $\sqrt{n_{p}}$ of the condition number of the optimally scaled version of $P$ [15].) Note that $\kappa(P)$ and $\alpha_{G, K}$ exhibit a general tendency to increase as either $\nu \rightarrow 0$ or $n$ increases, with $\kappa(P)$ being particularly volatile.

Now consider the data of Table 1 for the block triangular preconditioner. Several trends are apparent:

1. In the original formulation, the perturbations of both $\lambda=1$ and $\lambda \neq 1$ are increasing with $1 / \nu$. In the scaled formulation, the perturbations of $\lambda=1$ are insensitive to $\nu$. This behavior is consistent with the results of Theorem 3.2.

2. In the scaled formulation, the perturbations of $\lambda \neq 1$ show some growth with $1 / \nu$ for $n=32$, although there is no clear trend. This may derive from growth in the product $\kappa(P)\left\|(I-H)^{-1}\right\|_{2}$.

3. There is little or no increase in perturbation size (and a decrease in some cases) with the change of grid size from $n=16$ to $n=32$. This contrasts with the bounds from the analysis, which degrade as $h \rightarrow 0$.

Next, consider Table 2.

1. The perturbations of $\lambda \neq 1$ for both the unscaled and scaled problems show some growth with $1 / \nu$. The qualitative trends are similar, but the perturbations for the scaled formulations are larger. In contrast, the analysis (Theorem 4.2) suggests that the scaled version would be smaller; therefore it appears that the upper bounds of this analysis are not giving a complete indication of dependence on $\nu$.

2. The perturbations of $\lambda=1$ are insensitive to $\nu$ and there is essentially no difference between the perturbations of the eigenvalues with positive and negative real parts. As noted in Remark 4.1, we believe the dependence on $\alpha_{G, K}$ is an artifact of the proof.

3. The dependence on the mesh size is more pronounced for the triangular preconditioning, although there is no consistent pattern.

Thus, the analysis gives upper bounds on perturbation sizes, although it is not possible to completely correlate the analytic bounds and experimental results. This 
TABLE 5

Secant of largest principal angle between Range $\left(G^{T}\right)$ and Range $\left(K^{T}\right)\left(\alpha_{G, K}\right)$.

\begin{tabular}{c|cccccc}
$\nu$ & 1 & $1 / 10$ & $1 / 20$ & $1 / 30$ & $1 / 50$ & $1 / 100$ \\
\hline$n=16$ & 1.11 & 1.65 & 2.51 & 3.29 & 4.53 & 6.51 \\
$n=32$ & 1.16 & 1.72 & 2.76 & 3.77 & 5.57 & 8.96
\end{tabular}

TABLE 6

Maximum normalized perturbations of eigenvalues of $H$.

\begin{tabular}{c|cccccc}
$\nu$ & 1 & $1 / 10$ & $1 / 20$ & $1 / 30$ & $1 / 50$ & $1 / 100$ \\
\hline$n=16$ & 3.8 & 4.7 & 2.4 & 2.4 & 3.2 & 5.3 \\
$n=32$ & 8.3 & 4.8 & 3.4 & 10.8 & 73.5 & 31.1 \\
$n=64$ & 28.4 & 226.4 & 134.1 & 61.7 & 130.8 & 402.3
\end{tabular}

is likely due to the lack of a clear pattern in the behavior of $\kappa(P)$ and $\left\|(I-H)^{-1}\right\|_{2}$, together with the use of norm inequalities throughout the analysis that are not necessarily tight. For example, the analysis combines bounds on matrix $l_{2}$-norms derived from the underlying differential operator with $l_{\infty}$ norms derived from Gerschgorin bounds. We suspect that the factor of $1 / h$ used to relate these quantities artificially inflates the dependence of the bounds on the mesh size, although we see no way to avoid introducing this term. In general, both the analysis and experiments indicate a tendency for perturbations of the eigenvalues different from 1 to increase with $1 / \nu$, but growth appears to be at worst linear and for the block triangular preconditioning it can be reduced by scaling.

Finally, Table 6 examines the sensitivity to perturbation of the eigenvalues of the reduced matrix $H$. The entries are

$$
\left(\max _{\hat{\lambda} \in \sigma(H+\epsilon E)}|\hat{\lambda}-\lambda|\right) / \epsilon
$$

The results also indicate that the perturbations increase as the viscosity decreases, and they display some growth as the number of mesh points increase, roughly like that for the block diagonal preconditioner displayed in Table 2. Note that the dependence on both $1 / \nu$ and $n$ is much less severe than that of $\kappa(P)$ shown in Table 3 . Once again, this stands in contrast with analytic bounds such as those obtainable from the Bauer-Fike theorem [7, p. 342], which suggests perturbations proportional to $\kappa(P)$.

7. Inexact inner iteration. We conclude by examining the effect of inexact computation of the action of $F^{-1}$. For brevity, we restrict our attention to the unscaled version of $\mathcal{A}_{T}$; similar analysis leads to essentially the same conclusions for $\mathcal{A}_{D}$. If the action of $F^{-1}$ is approximated using an iterative method for each system $F w=v$, then $F^{-1}$ can be replaced by $F^{-1}+E$ in $(9)$. The perturbed preconditioned matrix is then $\mathcal{A}_{T}+\mathcal{E}$ where

$$
\mathcal{E}=\left(\begin{array}{ll}
F E & F E K^{T} \\
B E & B E K^{T}
\end{array}\right)
$$


For this analysis it will be useful to consider the complete version of (24),

$$
\hat{\mathcal{E}}_{T}=\left(\begin{array}{cc}
F E\left(I+K^{T}(I-H)^{-1} G\right) & F E K^{T} P \\
P^{-1}\left(I-(I-H)^{-1}\right) B E & P^{-1}\left(I-(I-H)^{-1}\right) B E K^{T} P \\
\cdot\left(I+K^{T}(I-H)^{-1} G\right) &
\end{array}\right)
$$

Suppose the approximate solution $\tilde{w}$ satisfies

$$
\frac{\|v-F \tilde{w}\|}{\|v\|} \leq \tau
$$

for some tolerance $\tau$. Standard inequalities yield the bounds on the relative error

$$
\frac{\|w-\tilde{w}\|}{\|w\|} \leq\|F\|\left\|F^{-1}\right\| \tau, \quad \frac{\|w-\tilde{w}\|}{\|w\|} \leq\|F\|\|E\| .
$$

Treating these as approximate equalities gives $\|E\| \approx \tau\left\|F^{-1}\right\|$, it follows that

$$
\begin{aligned}
& \left\|\hat{E}_{11}\right\| \leq \approx \tau\|F\|\left\|F^{-1}\right\|\left(1+\left\|K^{T}\right\|\left\|(I-H)^{-1}\right\|\|G\|\right), \\
& \left\|\hat{E}_{12}\right\| \leq \approx \tau\|F\|\left\|F^{-1}\right\|\left\|K^{T}\right\|\|P\| \text {, } \\
& \left\|\hat{E}_{21}\right\| \leq \approx \tau\left\|P^{-1}\right\|\left(1+\left\|(I-H)^{-1}\right\|\right)\|B\|\left\|F^{-1}\right\|\left(1+\left\|K^{T}\right\|\left\|(I-H)^{-1}\right\|\|G\|\right), \\
& \left\|\hat{E}_{22}\right\| \leq \approx \tau \kappa(P)\left\|I-(I-H)^{-1}\right\|\left\|F^{-1}\right\|\left\|K^{T}\right\| \text {. }
\end{aligned}
$$

Using the $l_{2}$-norm, let us consider the dependence of these bounds on the viscosity $\nu$ under the assumption (derived from the experimental results presented above) that the influence of $P$ and $H$ is not significant. The bounds on $\left\|\hat{E}_{11}\right\|,\left\|\hat{E}_{21}\right\|$, and $\left\|\hat{E}_{22}\right\|$ include the product $\left\|K^{T}\right\|\|G\|=O(\nu) \cdot O(1 / \nu)$, which is independent of $\nu$. Using the coercivity and continuity of the convection-diffusion operator, it can be shown that as functions of $\nu$,

$$
\|F\|=O(1), \quad\left\|F^{-1}\right\|=O(1 / \nu) .
$$

(An algebraic proof can be found in [4, Theorem 1].) This implies that the bounds on $\left\|\hat{E}_{11}\right\|$ and $\left\|\hat{E}_{21}\right\|$ grow as $1 / \nu$ decreases, whereas the bounds on $\left\|\hat{E}_{12}\right\|$ and $\left\|\hat{E}_{22}\right\|$ are independent of $\nu$. If $\tau$ is small, then as in $\S 3$ we can restrict our attention to the block diagonal entries, which indicates that the perturbations of eigenvalues different from 1 in this particular case do not depend on $\nu$. On the other hand, if $\tau$ is large, then it is not possible to exclude $\hat{E}_{21}$ from the Gerschgorin analysis, and the presence of $\left\|F^{-1}\right\|$ in this bound suggests that perturbations in all eigenvalues grow like $1 / \nu$.

Consider the implication of these observations on the performance of iterative methods for solving the discrete Oseen equations (3). We demonstrated in [3] that the iteration counts of Krylov subspace methods such as GMRES with the preconditioner $\mathcal{Q}_{T}(7)$ are independent of the mesh size, but that there is some deterioration in performance as $\nu$ decreases. Now suppose an inner iteration with stopping criterion (53) is used to approximate the action of $F^{-1}$. The analysis given here suggests that if $\tau$ is large, there may be additional degradation of performance for small $\nu$. In contrast, if $\tau$ is small then only $\lambda=1$ is sensitive to perturbation, which suggests that (extra) degradation of the inner iteration with decreasing $\nu$ may not be as pronounced. Figure 
FIG. 2. Iterations of FGMRES with inexact convection-diffusion solves.
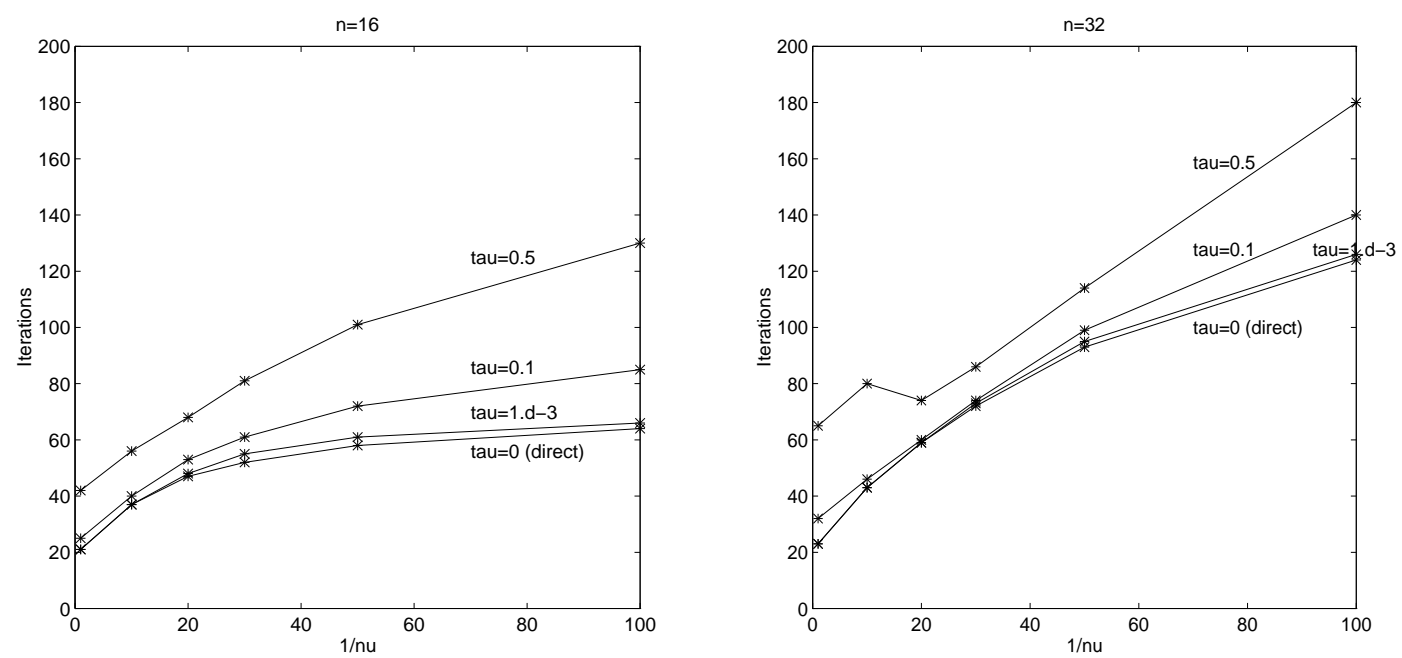

2 shows the results of numerical experiments that corroborate these observations. The figure plots iteration counts of right-preconditioned "flexible" GMRES (FGMRES) [12] as a function of $\nu$, for solving the discrete problem (3). The inner iteration for the convection-diffusion subproblems $F w=v$ was a line Gauss-Seidel method with stopping criterion (53). ${ }^{5}$ The test matrices were those used in $\S 6$; the right hand side $f$ consisted of normally distributed random numbers with mean 0 and variance 1 . The outer iteration used a zero initial guess and was stopped when the relative residual in the Euclidean norm was less than or equal to $10^{-6}$. The results with inner iteration are compared with using a direct method for the action of $F^{-1}$. They indicate that for the relatively modest tolerance $\tau=10^{-3}$, the inexact inner solves lead to little increase in outer iterations for any $\nu$. For less stringent $\tau$, additional outer iterations are required and the number of additional iterations becomes larger as $\nu$ decreases.

REMARK 7.1. It can be shown that for small $\tau$ the perturbations of the eigenvalues of the scaled system behave in the same way as the those for the unscaled system. However, scaling affects the relative weighting given to the two block equations of (3), which in turn may affect the iterative solver. Therefore, we have restricted our attention here to the unscaled system.

Acknowledgements. The author acknowledges helpful discussions with Santiago Arteaga, Luca Pavarino and Pete Stewart, and insightful comments from Hans Weinberger. The numerical results with FGMRES used software produced by Tim Kelley [11].

\section{REFERENCES}

[1] F. Brezzi and M. Fortin. Mixed and Hybrid Finite Element Methods. Springer-Verlag, New York, 1991.

\footnotetext{
${ }^{5}$ FGMRES allows the preconditioner to vary during the course of the iteration; it was used here because the number of inner iterations was not generally constant, i.e., the preconditioner is not a fixed linear operator. FGMRES is equivalent to GMRES when a direct solve is used for $F^{-1}$.
} 
[2] A. Brooks and T. Hughes. Streamline upwind/Petrov-Galerkin formulations for convection dominated flows with particular emphasis on the incompressible Navier-Stokes equations. Comp. Meth. Appl. Mech. Engng., 32:199-259, 1982.

[3] H. Elman and D. Silvester. Fast nonsymmetric iterations and preconditioning for Navier-Stokes equations. SIAM J. Sci. Comput., 17:33-46, 1996.

[4] H. C. Elman and M. H. Schultz. Preconditioning by fast direct methods for nonselfadjoint nonseparable elliptic problems. SIAM J. Numer. Anal, 23:44-57, 1986.

[5] R. Freund and N. M. Nachtigal. QMR: a quasi-minimal residual method for non-Hermitian linear systems. Numer. Math., 60:315-339, 1991.

[6] R. Glowinski. Numerical Methods for Nonlinear Variational Problems. Springer-Verlag, New York, 1984.

[7] G. H. Golub and C. F. Van Loan. Matrix Computations. The Johns Hopkins University Press, Baltimore, second edition, 1989.

[8] M. Gunzburger. Finite Element Methods for Viscous Incompressible Flows. Academic Press, San Diego, 1989.

[9] C. Johnson. Numerical Solution of Partial Differential Equations by the Finite Element Method. Cambridge University Press, New York, 1987.

[10] O. A. Karakashian. On a Galerkin-Lagrange multiplier method for the stationary Navier-Stokes equations. SIAM. J. Numer. Anal., 19:909-923, 1982.

[11] T. Kelley. Iterative Methods for Linear and Nonlinear Equations. SIAM, Philadelphia, 1995.

[12] Y. Saad. A flexible inner-outer preconditioned GMRES algorithm. SIAM J. Sci. Comput., 14:461-469, 1993.

[13] Y. Saad and M. H. Schultz. GMRES: A generalized minimal residual algorithm for solving nonsymmetric linear systems. SIAM J. Sci. Stat. Comput., 7:856-869, 1986.

[14] J. Stoer and R. Bulirsch. Introduction to Numerical Analysis. Springer-Verlag, New York, second edition, 1993.

[15] A. van der Sluis. Condition numbers and equilibration of matrices. Numer. Math., 14:14-23, 1969.

[16] R. Verfürth. A combined conjugate gradient-multigrid algorithm for the numerical solution of the Stokes problem. IMA J. Numer. Anal., 4:441-455, 1984.

[17] A. J. Wathen. Realistic eigenvalue bounds for the Galerkin mass matrix. IMA J. Numer. Anal., $7: 449-457,1987$.

[18] J. H. Wilkinson. The Algebraic Eigenvalue Problem. Oxford University Press, Oxford, 1965. 